\title{
Electronic Transport Through a Kink in an Electron Waveguide
}

\author{
M. Cemal Yalabik, Member, IEEE
}

\begin{abstract}
The current-voltage dependence corresponding to electronic transport through a kink in an electronic waveguide is analyzed. No phase breaking dissipation mechanisms are considered, but the effects̀ of the Coulomb interaction are included through a self consisterit approximation. The results indicate very nonlinear transport properties, including negative differential resistance and bistability.
\end{abstract}

\section{INTRODUCTION}

$\mathbf{N}$ ONLINEAR phenomena associated with the transport of electrons in mesoscopic structures has attracted considerable experimental as well as theoretical interest. (For a necessarily incomplete sampling of the relevant work in the area, see [1]-[24].) In this study, the current-voltage characteristics corresponding to the flow of electrons in a two dimensional electron waveguide with a kink, in the absence of any phase breaking dissipative interactions is presented. The results indicate that this geometry is expected to have very nonlinear transport characteristics at low temperatures, including a negative differential resistance at low voltages and a bistability, which may survive to room temperature for sufficiently small feature sizes. The negative differential resistance appears due to a "window" in the energy of electrons transmitted through the geometry. This window is not due to a "cavity" resonance that is typical of some quantum devices, but arises due to a broad wavelength selection process in electron transport. A consequence of this is much smaller charging effects and the expectation of a faster response.

First, the results in the absence of any Coulomb interaction will be reported. Then, the effects of the Coulomb interaction will be discussed within the framework of an approximate self-consistent model.

\section{THE MODEL}

One of the simplest structures one can consider as a deformation of a straight, narrow electron path is a kink of the form shown in Fig. 1. Even this simple geometry results in a rich structure in the transport properties. The resonances that are the result of quasi-standing waves that may be excited in the central part of this geometry lead to enhancements and suppressions of the current at various energies, leading to very nonlinear current-voltage characteristics.

Manuscript received May 5, 1993. The review of this paper was arranged by Associate Editor J. R. Hauser.

The author is with the Department of Physics, Bilkent University, 06533 Ankara, Turkey.

IEEE Log Number 9404375
At some low energies, the electron wavefunction may "bounce" from one side of the kink to the other and continue along the channel on the opposite side with little reflection. A "window" of energies for such transport exists, because with increasing forward momentum, the wavefunction will not be able to spend in the kink region a sufficient amount of time to permit the lateral shift in its position. (Numerical simulations of the time dependent behaviors of wave packets traveling through this structure display this property quite well.) This "window" results in a negative differential resistance at low voltages across the structure.

At higher energies, sharper resonances in the same nature as the Fano resonances in atomic and nuclear physics appear. These resonances correspond to cavity type oscillations of the wavefunction with a much looser coupling to the incoming and outgoing electron paths. Such resonances tend to have an amplitude in the cavity that builds up and decays with some relatively large time constant (in comparison to transit time), and therefore may collect an appreciable amount of charge. The mechanism in the resonant tunneling diode is an example to this. The inclusion of the effects of this charge leads to bistabilities in the current-voltage characteristics. In contrast to this, the fast transport mechanism described in the previous paragraph does not lead to appreciable charging or bistability. (A time dependent study of transport through this geometry is also under way, and will be reported separately.)

From elementary quantum mechanics, it is known that the wavefunction of an electron traveling in the $x$ direction in a rectangular waveguide is given by

$$
\Psi(x, y)=\sum_{n=-\infty}^{\infty} c_{n} e^{\imath k_{n} x} \sin \frac{n \pi y}{w}
$$

where $c_{n}$ are the expansion coefficients, $w$ is the width (in the $y$ direction) of the rectangular channel, and the wave number $k_{n}$ is related to the energy $E$ of the particle through

$$
E=\frac{\hbar^{2}}{2 m^{*}}\left(k_{n}^{2}+n^{2} \pi^{2} / w^{2}\right)
$$

Here, $m^{*}$ is the effective mass of the particle. (It is assumed that the electrons are sufficiently confined to the plane of the figure so that quantization in the $z$ direction results in energy bands well separated in energy.) Note that the kink geometry in Fig. 1 involves three rectangular regions with the parameter $w$ equal to $a, 2 a$, and again $a$. The problem of determining the transmission coefficients through the structure then involves the determination of the total wavefunction of the system by matching the solutions of the form of (1) at the boundaries of 


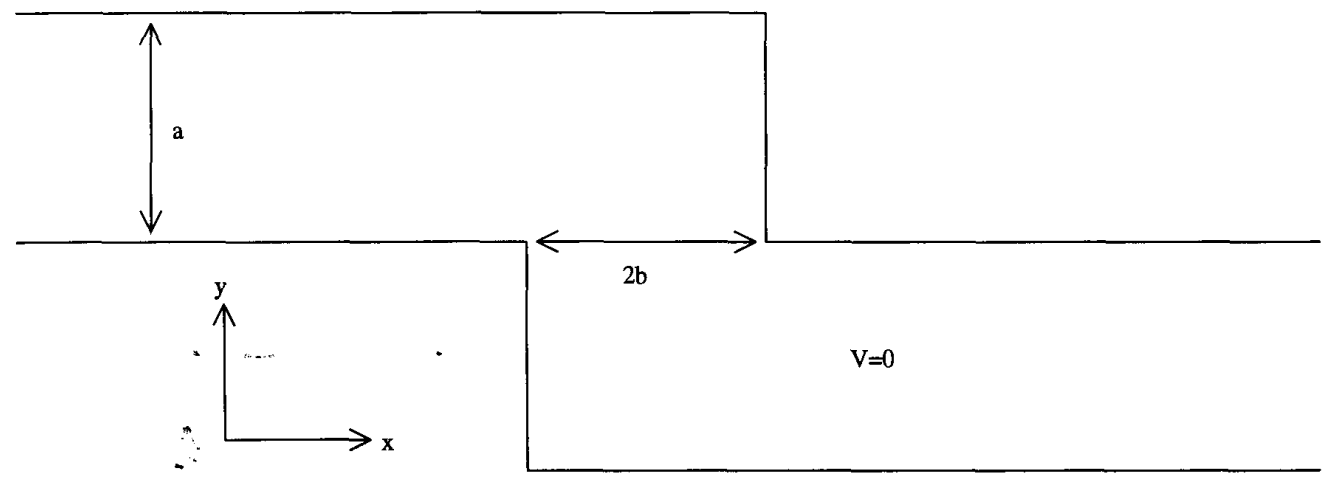

Fig. 1. The geometry analyzed in this work. The potential inside the kink shaped region is assumed to be zero, and infinitely large elsewhere. The left and right sides of the channel are connected to the contacts.

the three rectangular regions. The projections of the matching equations for the wave functions and their $x$ derivatives at the boundaries on functions of the form $\sin n \pi y / w$ result in a set of linear relationships among the expansion coefficients $c_{n}$. These equations then can be solved with assignments of values to coefficients that correspond to incident waves. In all of the results reported in this work, the summation in (1) was truncated to $|n| \leq 6$ for $w=a$ and to $|n| \leq 12$ for $w=2 a$. Obviously, for a specific value of the energy, not all of these "modes" carry a current (i.e., correspond to $k_{n}$ values which are real) in the narrow incoming and outgoing channels. However, one needs to include a sufficient number of these modes to maintain the accuracy in the wavefunction.

Neglecting the effects of the interparticle Coulomb interaction, and hence assuming that the current may be calculated through a superposition of the effects of single particle solutions of the Schrödinger equation through the two terminal Landauer formula [1], one obtains:

$$
I_{L \rightarrow R}=\frac{e \hbar}{\pi m^{*}} \sum_{n} \int_{k_{n}^{\min -L}}^{\infty} d k k T_{n}(k) F_{n}^{(L)}(k)
$$

Here, $I_{L \rightarrow R}$ is the electron current from left to right, $e$ is the electronic charge, $k^{\mathrm{min}-\mathrm{L}}$ is the minimum electron wavenumber for electrons incident from the left (a restriction that may arise due to the presence of a band edge at the contact), $T_{n}(k)$ is the transmission coefficient for electrons incident with mode number $n$ and wavenumber $k$, and $F^{(L)}(k)$ is the Fermi distribution at the left side contact. The subscript $n$ indicates that the associated quantity depends on the mode. The total current through the structure then is the difference of the electron currents in two directions: $I=I_{L \rightarrow R}-I_{R \rightarrow L}$.

Fig. 2 indicates the total transmission coefficient for electrons incident from one side of the structure (in the first subband) with energy $E$, and being transmitted on the other side in any mode. A natural parameter that scales the potential in this problem is $V_{0}$, defined through the relation $e V_{0}=$ $\hbar^{2} /\left(2 m^{*} a^{2}\right)$. (For a $10 \mathrm{~nm}$ wide channel and an effective mass corresponding to that in GaAs, $V_{0}=5.7 \mathrm{mV}$.) The zero of the energy has been chosen to be the lowest energy that will carry a current in the channels, i.e., the bottom of the lowest subband. Since the subband energy minima are given

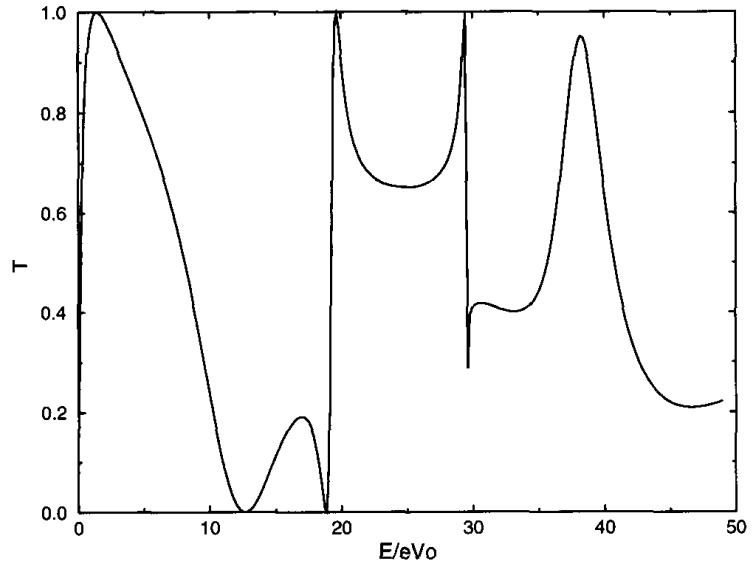

Fig. 2. The total transmission coefficient as a function of scaled energy. Note the "window" at low energies.

by $\epsilon_{n}=e V_{0} \pi^{2} n^{2}$, the second subband starts to carry current when $E / e V_{0} \approx 30$. Structure corresponding to this energy is apparent in the figure. The third subband starts to carry current when $E / e V_{0} \approx 79$. In all of the results to be presented, the electrons are taken to be incident from either side in the first subband, and may be reflected or transmitted through in the first subband, or the second subband if they have sufficient energy. (The incident electrons will be taken to have a Fermi energy $10 \mathrm{eV}_{0}$ above the first subband energy minima, limiting them to the first subband. However, they may be transmitted out in the second subband if the potential difference between the two sides is sufficiently large.)

Fig. 2 (as well as the other results that will be reported below) corresponds to a kink with $b / a=0.6$. Note that there is considerable structure in the transmission coefficient corresponding to the bouncing of the electron in the central region of the kink. The initial wide peak is the transmission process described above for motivating this study. The peaks and dips at higher wave numbers can be identified with energies at which a new mode starts to carry current in the central or extremal parts of the geometry. At even larger wave-number values (not shown in the figure), one obtains a 


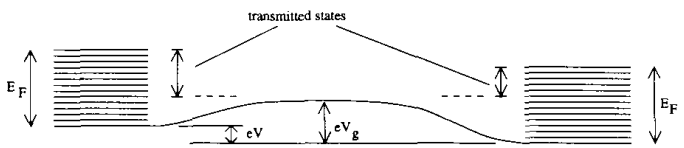

Fig. 3. The potential energy model used in the transport calculation. The central flat part of the potential is assumed to contain the kink.

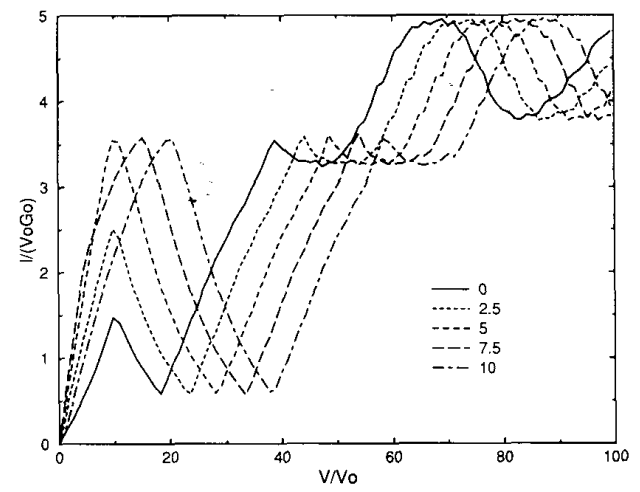

Fig. 4. The zero temperature current through the structure as a function of the voltage for various values of the potential at the central region $\left(\mathrm{V}_{g}\right.$ in Fig. 3). The legend identifies this potential for each curve in units of $V_{0}$.

repeating pattern of similar resonances with an approximately exponentially decreasing amplitude, corresponding to higher order standing waves generated in the central region.

In anticipation of the effects of the Coulomb interaction, the current has been calculated for an energy diagram as indicated in Fig. 3. It will be assumed that a smoothly varying electrostatic potential (which is fairly constant in a region of space which includes the kink) exists between the two contacts to this structure. Although the motivation for the inclusion of this central potential is to study the charging effects, at this point it may be taken to be the effect of an external gate. It will further be assumed that all electrons which have sufficient energy to reach the central kink region will be transmitted there with probability 1 , otherwise they will be fully reflected. The electrons then will be transmitted through the kink (which is assumed to be at a constant potential) with the same transmission probabilities (corresponding to their new kinetic energies) computed in the absence of the electrostatic potential. Once the electron passes through the kink, it will again be assumed to be transmitted to the second contact without reflection if it has sufficient energy to reach there and a state exists there to accommodate it. In effect, this approximation amounts to the neglect of the mechanics associated with the ramps indicated in Fig. 3, which summarizes the model. These approximations then allow the use of the transmission coefficients calculated above (in the absence of the electrostatic potential) in the estimation of transport properties in the presence of a flat potential at the kink region.

Fig. 4 shows the current through the structure as a function of the voltage between the two sides, assuming that the electronic density at the contacts may be represented by a Fermi distribution at zero temperature. (The Fermi energy was taken to be $E_{F}=10 \mathrm{eV}_{0}$ above the first subband energy minimum.) The family of curves were generated for a set of central region voltages. Note that the current through the structure decreases once the set of incident states (with an energy width of $10 e V_{0}$ ) exceed the energy window (again of width $10 e V_{0}$ ) leading to a negative differential resistance effect in the low voltage region. (The voltage difference across the full structure is twice the symmetric Fermi level shift at each side.) Larger central region potentials lead to a shift of this phenomena to larger voltages, but the maximum of the current is also enhanced due to smaller reverse current. At larger voltages across the structure, one obtains a current that is a function of the overlap of the $10 \mathrm{eV}$ wide incident electron states with the transmission characteristics given in Fig. 2 . The current plateau starting at $V / V_{0} \approx 40$ and the current peak at $V / V_{0} \approx 70$ may be associated with the transmission plateau and peak at $E / \mathrm{eV}_{0} \approx 20$ and 38 , respectively. Since there is practically no current flowing in reverse direction under such large biases, a shift in the central region voltage merely serves to shift the current-voltage curve.

\section{The Charging EfFects}

Obviously, in order to obtain a realistic description of the properties of an actual experimental implementation of this geometry, one would have to include various effects, including the shape of the potential profile at the walls, the actual potential distribution that ends up on an actual material, the change in the potential due to the presence of other electrons, as well as interelectronic and other scattering mechanisms. Note however that the important physical feature that drives the nonlinearity in transport is the translation of the particles in a direction perpendicular to the current flow in a relatively short distance scale. The sharpness of the potential profile is therefore not expected to be too critical since one expects low particle densities for a "resonant" electron at the corners of the geometry.

Now, a model will be presented in which the effects-of the Coulomb interaction can be included in a self consistent, but simplistic way: It will be assumed that the electrostatic potential due to the electrons present in the channel is relatively smooth, and can be modeled by a corresponding energy diagram as shown in Fig. 3. (A number of potential distributions corresponding to electron densities associated with the current levels indicated in Fig. 4 were calculated and were found to be consistent with this approximation, as would be expected due to the smoothing out property of the Poisson equation.) It can now be assumed that the additional potential at the flat central part of Fig. 3 is proportional to the amount of charge in this region. Again, the mechanics and charging effects associated with the ramps in Fig. 3 are neglected.

At this point, it should be pointed out that the structure has a bound state, with an energy $2.6 \mathrm{e} V_{0}$ below the lowest energy current carrying state. Solution of the Poisson equation for the charge density corresponding to an electron in this bound state indicates that this electron results in an increase in the potential of approximately $1 L_{11} / 1{ }^{\prime} 11::::^{-}-$. It will therefore be assumed that each electror : - he contral region of the 


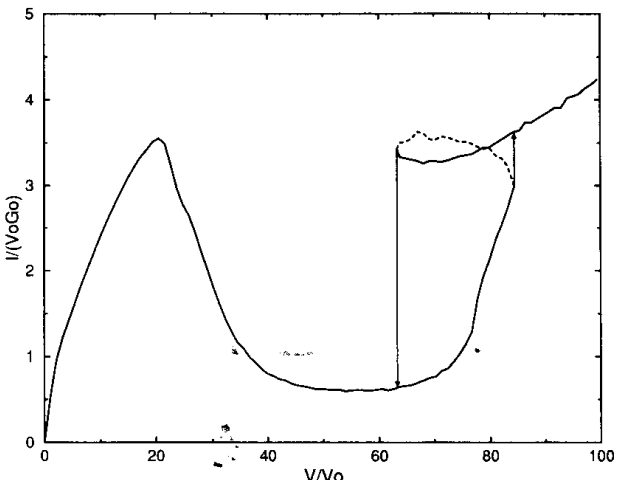

(a)

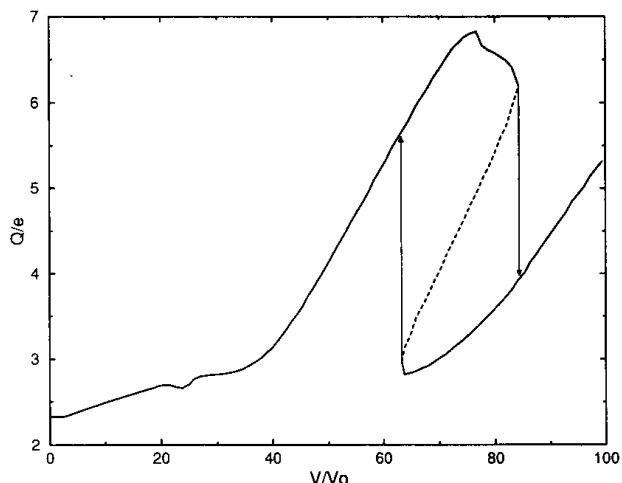

(b)

Fig. 5. Results of the self consistent calculation with the charging effects. The zero temperature current (a) and the charge in the central region (b) as a function of the voltage. Note the small amount of charging at low voltages due to fast transit of electrons.

kink contributes approximately this amount to the potential. Although this model is admittedly crude, it should nevertheless provide an insight into the effects of the Coulomb interaction.

The self-consistent current-voltage characteristic is then computed as follows: For each value of the potential difference, a value for the potential at the position of the kink ( $V_{g}$ in Fig. 3) is assumed. The expansion coefficients of the wave functions corresponding to the incident energy levels are then calculated, and the total particle density is obtained by a superposition of all of the modes that contribute to charge transport. (Obviously, for states which can transport electrons in and out freely, there is a small contribution to particle density, while states that trap electrons into resonances yield much larger contributions.) This procedure then yields a certain value for the amount of charge in the kink region. The assumed value of $V_{g}$ is modified and the above procedure is repeated until the assumed value of this potential is consistent with the assumption that it should be equal to $4 V_{0}(a / 10 \mathrm{~nm})^{-1}$ per electronic charge. Obviously, at voltages where one finds bistability, there is more than one solution to this procedure.

Fig. 5(a) shows the current-voltage characteristics, while Fig. 5(b) shows the charge in the central region as a function of the voltage for an $a=10 \mathrm{~nm}$ structure. Note the negative differential resistance as well as the bistability at

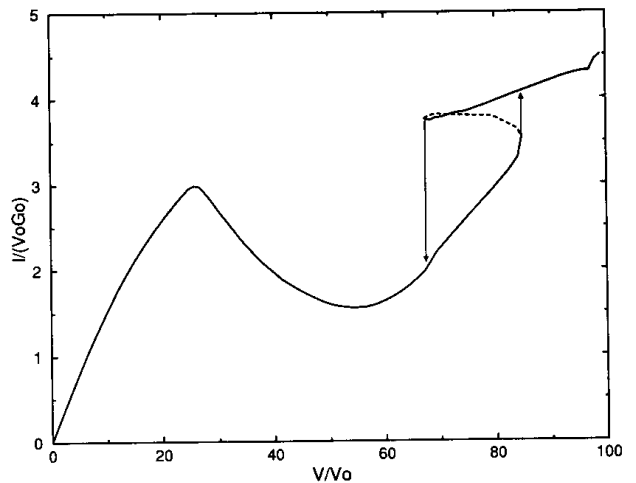

(a)

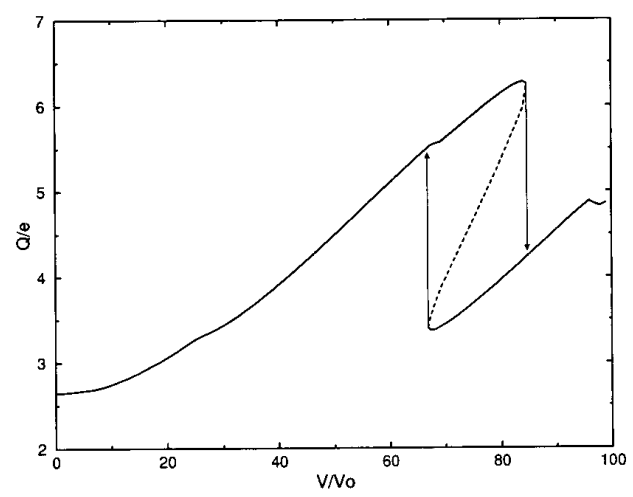

(b)

Fig. 6. The variables in Fig. 5 calculated for contact Fermi distributions with a temperature $T$ such that $k_{B} T=4.5 \mathrm{eV}$.

larger voltages. Fig. 5(b) indicates that the charge in the kink region does not change appreciably throughout the negative differential resistance region due to the fast transit of the electrons at these energies. (Whatever charge that does exist however, results in a characteristics that correspond to some central region potential as a comparison of Fig. 5(a) with Fig. 4 indicates.) Once the electron energies start to reach $E \approx 20 e V_{0}$ (corresponding to $V \approx 40 V_{0}$ ), appreciable charge buildup starts at the corresponding sharp resonances, and the current is suppressed. As is typical for sharp resonances of this type, at higher potential differences a second stable (and much smaller) value for the amount of charge is possible, resulting in a larger current (of the scale indicated in Fig. 4). The large current (less trapping of charge) is then self-consistent with the small charge. The bistability in the resonant tunneling diode is of a similar nature.

Fig. 6 displays the same variables with the Fermi distributions at the two contacts modified for a temperature that corresponds to $k_{B} T=4.5 \mathrm{eV}_{0}$, (where $k_{B}$ is the Boltzmann constant and $T$ is the temperature), which for an $a=10 \mathrm{~nm}$ structure corresponds to $T=300 \mathrm{~K}$.

\section{CONCLUSIONS}

It is apparent that even a very simple structure as a kink in a straight electron waveguide yields very interesting transport 
properties. Negative differential resistance and bistabilities have some potential for applications, and these features would be expected to appear in an experimental realization of this structure, although it would be simplistic to expect accurate quantitative agreement with the results of the idealized model presented here. The sharpness of the potential pattern is not expected to play a fundamental role, main features of the current-potential difference characteristic should appear as long as the electron path widens and is displaced in a direction perpendicular to the direction of current flow in a distance scale comparable to the width of the channels.

Work is in progress to study the dynamics of the charge buildup, and the switching process in the bistable region. The methodology to be developed for such studies should have potential for application to similar structures.

\section{REFERENCES}

[1] L. Landauer, "Spatial variations of currents and fields due to localized scatterers in metallic conduction," IBM J. Res. and Dev., vol. 1, pp. 223-228, 1957

[2] M. Büttiker, "Four-terminal phase-coherent conductance," Phys. Rev. Lett., vol. 57, no. 14, pp. 1761-1764, 1986.

[3] G. Timp, H. U. Barranger, P. de Vegvar, J. E. Cunningham, R. E. Howard, R. Behringer, and P. M. Mankiewich, "Propagation around a bend in a multichannel electron waveguide," Phys. Rev. Lett., vol. 60 , no. 20 , pp. 2081-2084, 1988.

[4] D. A. Wharam, T. J. Thornton, R. Newbury, M. Pepper, H. Ahmed, J. E. Frost. D. G. Hasko, D. C. Peacock, D. A. Ritchie, and G. A. C. Jones, "One-dimensional transport and the quantization of the ballistic resistance," J. Phys. C: Solid State Phys., vol. 21, pp. L209-L214, 1988.

[5] D. A. Wharam, M. Pepper, H. Ahmed, J. E. F. Frost, D. G. Hasko, D. C. Peacock, D. A. Richie, and G. A. Jones, "Addition of the onedimensional quantized ballistic resistance," J. Phys. C: Solid State Phys., vol. 21, pp. L887-L891, 1988.

[6] B. J. van Wees, H. van Houten, C. W. J. Beenakker, J. G. Williamson, and C. T. Foxon, "Quantized conductance of point contacts in a twodimensional electron gas," Phys. Rev. Lett., vol. 60, no. 9, pp. 848-850, 1988

[7] C. J. B. Ford, S. Washburn, M. Büttiker, C. M. Knoedler, and J. M. Hong, "Influence of geometry on the Hall Effect in ballistic wires," Phys. Rev. Lett., vol. 62, no. 23, pp. 2724-2727, 1989.

[8] C. W. J. Beenakker and H. van Houten, "Magnetotransport and nonadditivity of point-contact resistances in series" Phys. Rev B, vol. 39 , no. 14. pp. $10445-10448,1989$.

[9] Y. Avishai and Y. B. Band, "Quantum electronic conductance of a terminal junction," Phys. Rev. Lett., vol. 62, no. 21, pp. 2527-2530, 1989.

[10] C. W. J. Beenaker and H. van Houten, "Billiard model of a ballistic multiprobe conductor," Phys. Rev. Lett., vol. 63, no. 17, pp. 1857-1860, 1989.
[11] A. Szafer and A. D. Stone, "Theory of quantum conduction through a constriction," Phys. Rev. Lett., vol. 62, no. 3, pp. 300-303, 1989.

[12] Y. Takagaki and D. K. Ferry, "Quantum transmission in a junction of crossed wires with a box resonator," Phys. Rev. B, vol. 44, no. 15, pp. $8399-8402,1991$

[13] Y. Takagaki and D. K. Ferry, "Double quantum point contacts in series," Phys. Rev. B, vol. 45, no. 15, pp. 13494-13498, 1992.

[14] U. Ravaioli, T. Kerkhoven, M. Raschke, and A. T. Galick, "Numerical simulation of electron confinement in contiguous quantum wires," Superlattices and Microstructures, vol. 11, pp. 343-346, 1992.

[15] M. Field, C. G. Simth, M. Pepper, D. A. Ritchie, J. E. F. Frost, G. A. C. Jones, and D. G. Hasko, "Measurements of Coulomb blockade with a noninvasive voltage probe." Phys. Rev. Lett., vol. 70, no. 9. pp. $1311-1314,1993$

[16] N. F. Johnson and M. C. Payne, "Microscopic theory of periodic conductance resonances in narrow channels," Phys. Rev. Lett., vol. 70 , no. 10 , pp. 1513-1516, 1993.

[17] M. Leng and C. S. Lent, "Recovery of quantized ballistic conductance in a periodically modulated channel." Phys. Rev. Lett., vol. 71, no. 1 , pp.137-140, 1993.

[18] M. C. Yalabik, "Electronic transport in a laterally patterned resonant structure," in Negative Differential Resistance and Instabilities in 2-D Semiconductors, N. Balkan, B. K. Ridley, and A. J. Vickers, Eds. New York: Plenum, 1993, pp. 171-178.

[19] A. I. Onipko and I. V. Zozulenko, "Quantum interference effects in a II-shaped electron waveguide," Semicond. Sci. Technol., vol. 8, pp. $2115-2120,1993$

[20] F. Sols, M. Macucci, U. Ravaioli, and K. Hess, "Theory for a quantum modulated transistor," J. Appl. Phys., vol. 66, no. 8, pp. 3892-3906, 1989.

[21] J. C. Wu, M. N. Wybourne, W. Yindeepol, A. Weisshaar, and S. M. Goodnick, "Interference phenomena due to a double bend in a quantum wire," Appl. Phys. Lett., vol. 59, no. 1, pp. 102-104, 1991

[22] J C Wu M N. Wybourne, A. Weisshaar, and S M Goodnick "Waveguide effects in quantum wires with double-bend discontinuities," J. Appl. Phys, vol. 74. no. 7, pp. 4590-4597, 1993.

[23] Z. Ji, "Ballistic transport through a double bend in an electron waveguide." J. Appl. Phys., vol. 9, no. 1, pp. 4468-4472, 1993

[24] T. Kawamura and J. P. Leburton, "Quantum ballistic transport through a double-bend waveguide structure: effects of disorder," Phys. Rev', vol. B48, no. 12 , pp. $8857-8865,1993$

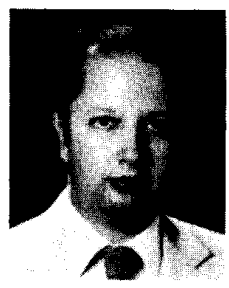

M. Cemal Yalabik (M'88) received the B.S. degree in electrical engineering from Middle East Technical University, Ankara, Turkey, in 1971, and the M.S. and $\mathrm{Ph} . \mathrm{D}$. degrees from Brown University. Providence, RI, in 1973 and 1976, respectively.

He worked at Temple University, Philadelphia, PA, during 1976-1978 and 1985-1987 and Middle East Technical University (1979-1985). In 1987 he joined Bilkent University, Ankara. Turkey. His research interests include quantum regime device simulation, quantum dissipation, and statistical physics. 